\title{
Treatment application of rivaroxaban in Chinese patients with livedoid vasculopathy
}

\author{
This article was published in the following Dove Press journal: \\ Journal of Pain Research \\ 17 March 2017 \\ Number of times this article has been viewed
}

\author{
Wenji Chen' \\ Lina Fan' \\ Yanyan Wang ${ }^{2}$ \\ Xiaohu Deng ${ }^{2}$ \\ 'Department of Rheumatology, \\ Hainan Branch of Chinese People's \\ Liberation Army General Hospital, \\ Sanya, ${ }^{2}$ Department of Rheumatology, \\ Chinese People's Liberation Army \\ General Hospital, Beijing, People's \\ Republic of China
}

\begin{abstract}
Livedoid vasculopathy (LV) is a chronic prothrombotic disease of cutaneous microcirculation resulting in cutaneous ischemia and infarction. As a rare disease, LV has an estimated incidence of ten cases per million. Not only correct diagnosis but also effective treatments are very difficult for patients with LV. Due to the lack of large-scale studies in this rare disease, LV poses a great challenge to the doctors, and existing treatment has always been an individual attempt with off-label application. The main goals in the treatment of patients with LV are to avoid the repeated occurrence of active cutaneous lesions and prevent painful ulceration and irreversible scarring. The current report describes the cases of three Chinese patients with LV receiving rivaroxaban treatment, an oral direct inhibitor of factor Xa inhibitor, and observes the treatment effect of rivaroxaban during the follow-up. As an injection-free alternative to lowmolecular-weight heparin (LMWP) and monitoring-free alternative to warfarin, rivaroxaban improves the quality of life and enhances the compliance of patients. All patients consider rivaroxaban as more tolerable than previous drugs and, therefore, continue the application of rivaroxaban, effectively improving the treatment effect of drugs and successfully avoiding the repeated occurrence of active cutaneous lesions. Treatment application of rivaroxaban in Chinese patients with LV successfully avoids the recurrence of active cutaneous lesions and prevents the progressive ulceration and scarring.
\end{abstract}

Keywords: livedoid vasculopathy, rivaroxaban

\section{Introduction}

Livedoid vasculopathy (LV) is a chronic prothrombotic disease of cutaneous microcirculation resulting in cutaneous ischemia and infarction. As a rare disease, LV has an estimated incidence of ten cases per million. ${ }^{1} \mathrm{LV}$ mostly appears in late adolescence up to the age of 30 years, and patients with LV have an average age of 45 years. ${ }^{2} \mathrm{LV}$ shows a distinct female preference and affects three times as many females as males. ${ }^{1}$ Not only correct diagnosis but also effective treatments are very difficult for patients with LV. ${ }^{3}$ Due to the lack of large-scale studies in this rare disease, LV poses a great challenge to the doctors, and existing treatment has always been an individual attempt with off-label application. The main goals in the treatment of patients with LV are to avoid the repeated occurrence of active cutaneous lesions and prevent painful ulceration and irreversible scarring. As an important result of recent data, LV has been defined as a coagulation disease and systemic anti-coagulant drugs, especially the rivaroxaban, have been shown to be effective as a treatment for patients with LV. ${ }^{4}$ As the first multicenter study, rivaroxaban for livedoid vasculopathy (RILIVA) trial has been published by Weishaupt et $\mathrm{al}^{5}$ and suggested that rivaroxaban is a suitable treatment option for
Correspondence: Xiaohu Deng Department of Rheumatology, Chinese People's Liberation Army General Hospital, Fuxing Road 28, Beijing 100853, People's Republic of China

Tel +86 I36 9123 5I55

Email dengxh1970@sina.com 
patients with LV. The current report describes the cases of three Chinese patients with LV receiving the treatment of rivaroxaban and observes the treatment effect of rivaroxaban during the follow-up. Written informed consents were provided by the three patients to have the case details and any accompanying images published.

\section{Case presentation \\ Case I}

Female patient, 38 years old, visited our hospital on November 17, 2014. She complained of repeated skin eruptions with sting and tenderness in both lower limbs for 4 months. Red skin eruptions did not fade under pressure, first having the appearance of tip, then gradually expanding and deeply bursting and finally coalescing and leaving the shrinkage and pigmentation, with high skin temperature (Figure 1A and C). She had no special medical history.

No abnormality was found by cardiopulmonary physical examination, and the electrocardiogram and chest X-ray were normal. There were no obvious edema and abnormal reflection in either lower limbs. Laboratory analyses revealed elevated levels of erythrocyte sedimentation rate (ESR, $24 \mathrm{~mm} / \mathrm{h}$ ), carbohydrate antigen 125 (CA125, $48.75 \mathrm{U} / \mathrm{mL}$ ), and CA724 (19.97 U/mL), normal levels of C-reactive protein (CRP), homocysteine, alanine aminotransferase (ALT) and creatinine, and negative antinuclear antibody, antineutrophil cytoplasm antibody, rheumatoid factor, tuberculosis antibody, autoantibody, and anticardiolipin antibody. Skin biopsy revealed neutrophils, lymphocytes, or plasmacytes in a few small vessel walls (Figure 2A). Meanwhile, not only no thrombus was seen in the vessel lumen, but no fibrinoid necrosis was noted in the vessel wall as well (Figure 2B).

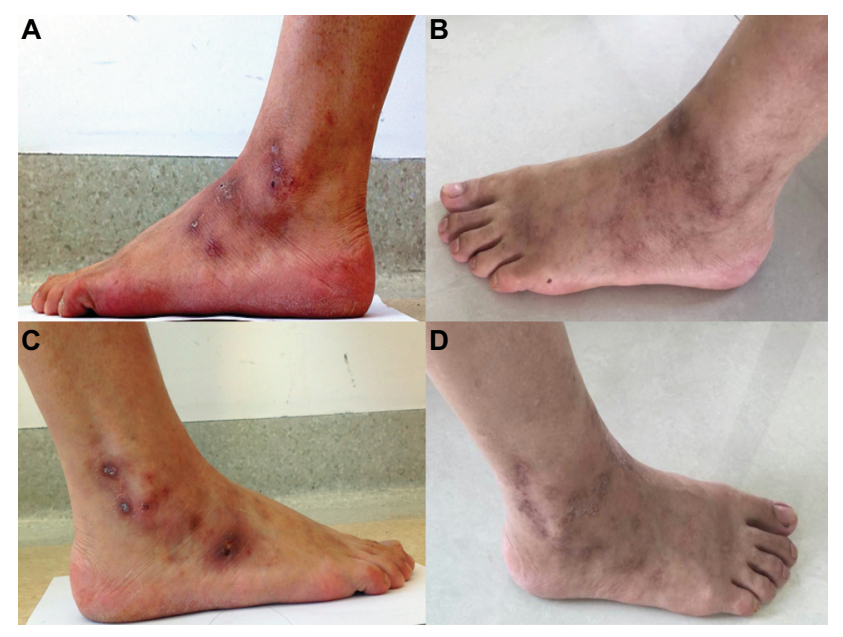

Figure I Case I: before the treatment of rivaroxaban (A and $\mathbf{C}$ ) and after the treatment of rivaroxaban (B and $\mathbf{D})$.

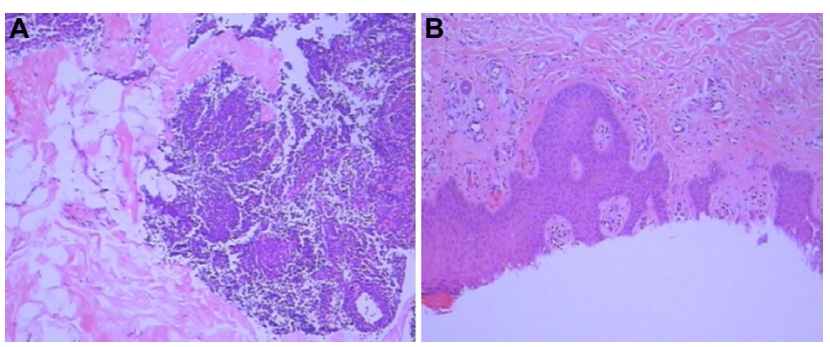

Figure 2 Case I: skin biopsy before treatment - neutrophils, lymphocytes, or plasmacytes in a few small vessel walls $(\mathbf{A})$ and no thrombus in vessel lumen and no fibrinoid necrosis in vessel wall (B).

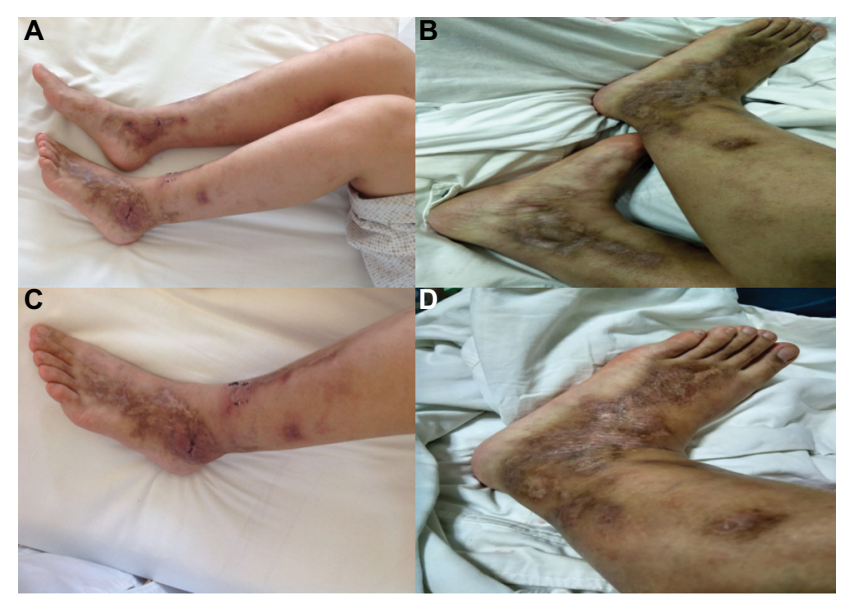

Figure 3 Case 2: before the treatment of rivaroxaban ( $\mathbf{A}$ and $\mathbf{C}$ ) and after the treatment of rivaroxaban (B and $\mathbf{D})$.

She used the glucocorticoid, immunosuppressant, and other externally used drugs, and skin eruptions appeared repeatedly. After being admitted to our hospital, glucocorticoid was gradually decreased and then finally ceased, immunosuppressant was ceased, and rivaroxaban $10 \mathrm{mg} / \mathrm{d}$ was regularly taken once a day. There were no new skin eruptions in either lower limbs. Skin eruptions disappeared after 3 months and had not recurred after 23 months (Figure 1B and D).

\section{Case 2}

Female patient, 30 years old, visited our hospital on July 9 , 2015. She complained of repeated skin eruptions with ulcerations and nodules for 4 years. Red skin eruptions first had the appearance of tip and then gradually expanded. For these skin eruptions, it was easy to burst but difficult to coalesce. There was obvious swelling with tenderness and high skin temperature around the skin eruptions. Scarring and pigmentation were formed due to the repeated appearance of skin eruptions (Figure 3A and C). She had medical history of chickenpox appearing at the same time of skin eruptions, 4 years ago.

Laboratory analyses showed normal levels of blood platelet count, ESR, CRP, ALT, and creatinine and negative autoantibody and tuberculosis antibody. Skin biopsy showed 
vascular proliferation in the dermis. There were neutrophils and lymphocytes around cutaneous appendages.

The patient used prednisone acetate, thalidomide, colchicine, and hydroxychloroquine. Due to the lack of treatment effect, prednisone acetate was ceased, and other drugs were not regularly used. After being admitted to our hospital, rivaroxaban was taken for 6 months, and skin eruptions had not recurred after 18 months (Figure 3B and D).

\section{Case 3}

Female patient, 39 years old, visited our hospital on October 9,2013 . She complained of repeated skin eruptions in both lower limbs for 2 years. Ulcerations and nodules appeared with effusion and scarring after skin eruptions burst. Skin eruptions were dark red with sting, tenderness, and high skin temperature (Figure 4A). She had medical history of urticaria and tuberculosis.

Swelling and pain were not seen, and movement was not restricted in lower limbs and all joints. Laboratory analyses showed elevated levels of white blood cell count $\left(11.88 \times 10^{9} / \mathrm{L}\right)$, blood platelet count $\left(353 \times 10^{9} / \mathrm{L}\right)$, ESR $(32 \mathrm{~mm} / \mathrm{h})$, CRP $(0.4 \mathrm{mg} / \mathrm{dL})$, CA125 (65.24 U/mL), and CA724 (13.13 U/ $\mathrm{mL}$ ) and normal levels of hemoglobin, ALT, creatinine, immunoglobulin, and complement. Skin biopsy showed chronic inflammation of cutaneous tissue. There were eosinophils in the superficial layer of dermis, inflammatory cells in vessel walls, and fibrinoid necrosis in a few vessel walls (Figure 5).

She used methylprednisolone, levofloxacin, and indomethacin, and skin eruptions recurred after ceasing methylprednisolone. Immunosuppressant, immunoglobulin, II recombinant human $\alpha$ receptor antibody fusion protein of tumor necrosis factor (rhRPTN), and adalimumab were also added to treat the skin eruptions. However, due to the recurrence of tuberculosis, methylprednisolone and immunosuppressant were ceased, and rivaroxaban was regularly taken for 3 months. Skin eruptions had not recurred after 23 months (Figure 4B).

\section{Discussion}

The pathogenesis of LV is cutaneous ischemia and infarction caused by the occlusion of capillary microcirculation. ${ }^{6}$

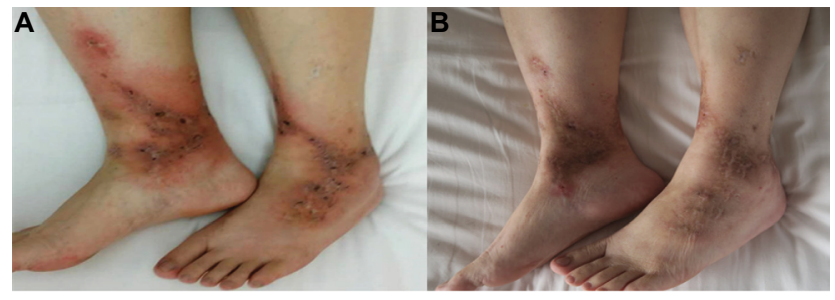

Figure 4 Case 3: before the treatment of rivaroxaban $(\mathbf{A})$ and after the treatment of rivaroxaban $(\mathbf{B})$.

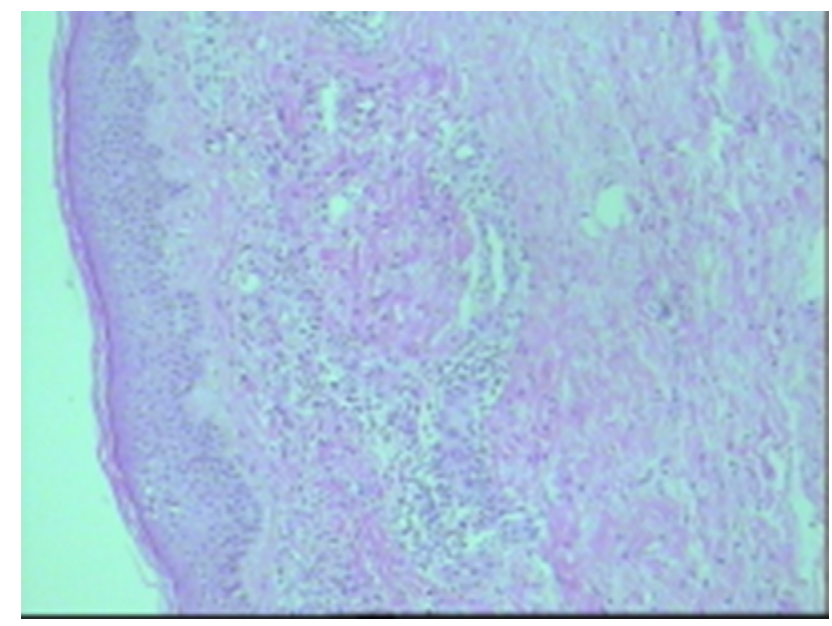

Figure 5 Case 3: skin biopsy before treatment shows eosinophils in the superficial layer of dermis, inflammatory cells in vessel walls, and fibrinoid necrosis in a few vessel walls.

Although there are LV-like changes in autoimmune and neoplastic diseases, they develop as secondary lesions. ${ }^{78}$ Perivascular inflammatory infiltration is secondary to the inflammation in an advanced stage and less prominent compared with coagulation function. ${ }^{9} \mathrm{LV}$ is almost exclusively located on both lower limbs, particularly the surrounding region of ankle and dorsal aspect of feet. Characteristic appearances of LV include one or more of triad livedo racemosa, ulceration, and atrophie blanche. Disturbed perfusion of cutaneous microcirculation leads to livedo racemosa, and the latter appears in a precursor stage to ulceration. The localized sensation of pain rather than fatigue appears in this stage and is of great importance as an early warning of continuously cutaneous ischemia and infarction with ulceration and scarring. Due to an inadequate blood supply to the cutaneous layers, ulceration develops as the result of cutaneous ischemia in the acute stage of LV. ${ }^{3}$ Atrophie blanche is an irreversible scarring representing the remnants of cutaneous infarction at the end of the restructuring process and accompanying postinflammatory pigmentation can be partially reabsorbed in the further stage.

The main goals in the treatment of patients with LV are to avoid the repeated occurrence of active cutaneous lesions and prevent the progressive ulceration. Effective treatment of LV is difficult, and limited options of drugs are available. In light of the coagulation disease, systemic anticoagulation drugs, such as low-molecular-weight heparin (LMWH) and warfarin, have been reported as treatment options. ${ }^{10}$ As has been the experience of European doctors, the treatment effect can be satisfactorily achieved for most patients with the application of LMWH. ${ }^{11,12}$ Based on previous literature, warfarin is also deemed as an option with the regular monitoring of international normalized ratio. ${ }^{13}$ However, LV is a 
chronic disease, and patients with LV complain about daily injections of LMWH and repeated monitoring of international normalized ratio. Meanwhile, the application of a fibrinolytic drug has strict indications due to severe risk of massive hemorrhages. ${ }^{14}$ Although experimental observation has also reported the benefit of immunoglobulin in patients with $\mathrm{LV}$, it inevitably results in economic burden on patients with LV. ${ }^{15}$

Recent data have suggested that rivaroxaban, an oral direct inhibitor of factor $\mathrm{Xa}$ inhibitor, has the potential to alleviate the intractable pain and cutaneous ischemia and prevent the occurrence of ulceration and scarring in patients with LV. ${ }^{16}$ As the first multicenter study, RILIVA trial has been published by Weishaupt et $\mathrm{al}^{5}$ and has suggested that rivaroxaban is a suitable treatment option for patients with LV. As an injection-free alternative to LMWH and monitoring-free alternative to warfarin, rivaroxaban improves the quality of life and enhances the compliance of patients. ${ }^{17}$ All patients consider rivaroxaban as more tolerable than previous drugs and, therefore, they continue the application of rivaroxaban, effectively improving the treatment effect of the drug and successfully avoiding the repeated occurrence of active cutaneous lesions.

\section{Conclusion}

The current report presented three Chinese cases with LV receiving rivaroxaban treatment and confirmed the treatment benefit of rivaroxaban in patients with LV during the follow-up. Treatment application of rivaroxaban in Chinese patients with LV successfully avoids the recurrence of active cutaneous lesions and prevents the progressive ulceration and scarring.

\section{Acknowledgment}

We are grateful to all participants for their participation in this work.

\section{Disclosure}

The authors report no conflicts of interest in this work.

\section{References}

1. Fritsch P, Zelger B. Livedo vasculitis. Hautarzt. 1995;46(3):215-224. quiz 222-223.

2. Goerge T, Weishaupt C, Metze D, et al. Livedoid vasculopathy in a pediatric patient with elevated lipoprotein(a) levels: prompt response to continuous low-molecular-weight heparin. Arch Dermatol. 2010;146(8):927-928.

3. Kerk N, Goerge T. Livedoid vasculopathy - current aspects of diagnosis and treatment of cutaneous infarction. J Dtsch Dermatol Ges. 2013;11(5): 407-410.

4. Kerk N, Drabik A, Luger T, Schneider SW, Goerge T. Rivaroxaban prevents painful cutaneous infarctions in livedoid vasculopathy. $\mathrm{Br} J$ Dermatol. 2013;168(4):898-899.

5. Weishaupt C, Strölin A, Kahle B, et al. Anticoagulation with rivaroxaban for livedoid vasculopathy (RILIVA): a multicentre, single-arm, open-label, phase 2a, proof-of-concept trial. Lancet Haematol. 2016; 3(2):e72-e79.

6. Callen JP. Livedoid vasculopathy: what it is and how the patient should be evaluated and treated. [comment]. Arch Dermatol. 2006;142(11): $1481-1482$

7. Marzano AV, Vezzoli P, Berti E. Skin involvement in cutaneous and systemic vasculitis. Autoimmun Rev. 2013;12(4):467-476.

8. Gonzalez-Santiago TM, Davis MD. Update of management of connective tissue diseases: livedoid vasculopathy. Dermatol Ther. 2012;25(2): 183-194.

9. Hairston BR, Davis MD, Pittelkow MR, Ahmed I. Livedoid vasculopathy: further evidence for procoagulant pathogenesis. Arch Dermatol. 2006; 142(11):1413-1418

10. Davis MD, Wysokinski WE. Ulcerations caused by livedoid vasculopathy associated with a prothrombotic state: response to warfarin. $J \mathrm{Am}$ Acad Dermatol. 2008;58(3):512-515.

11. Goerge T. Niedermolekulare Heparin-Therapie zur Behandlung der Livedovaskulopathie [Low-molecular-weight heparin for the treatment of livedoid vasculopathy]. Aktuelle Derm. 2010;36:484-487.

12. Sunderkotter C, de Groot K. Therapie von Vaskulitiden und Vaskulopathien [Therapy of vasculitides and vasculopathies]. Hautarzt. 2008; 59:382-393.

13. Browning CE, Callen JP. Warfarin therapy for livedoid vasculopathy associated with cryofibrinogenemia and hyperhomocysteinemia. Arch Dermatol. 2006;142(1):75-78.

14. Deng A, Gocke CD, Hess J, Heyman M, Paltiel M, Gaspari A. Livedoid vasculopathy associated with plasminogen activator inhibitor-1 promoter homozygosity $(4 \mathrm{G} / 4 \mathrm{G})$ treated successfully with tissue plasminogen activator. Arch Dermatol. 2006;142(11):1466-1469.

15. Kreuter A, Gambichler T, Breuckmann F, et al. Pulsed intravenous immunoglobulin therapy in livedoid vasculitis: an open trial evaluating 9 consecutive patients. J Am Acad Dermatol. 2004;51(4):574-579.

16. Perzborn E, Strassburger J, Wilmen A, et al. In vitro and in vivo studies of the novel antithrombotic agent BAY 59-7939 - an oral, direct factor Xa inhibitor. J Thromb Haemost. 2005;3(3):514-521.

17. Lassen MR, Ageno W, Borris LC, et al; RECORD3 Investigators. Rivaroxaban versus enoxaparin for thromboprophylaxis after total knee arthroplasty. N Engl J Med. 2008;358(26):2776-2786.
Journal of Pain Research

\section{Publish your work in this journal}

The Journal of Pain Research is an international, peer reviewed, open access, online journal that welcomes laboratory and clinical findings in the fields of pain research and the prevention and management of pain. Original research, reviews, symposium reports, hypothesis formation and commentaries are all considered for publication.

\section{Dovepress}

The manuscript management system is completely online and includes a very quick and fair peer-review system, which is all easy to use. Visit http://www.dovepress.com/testimonials.php to read real quotes from published authors. 